\title{
Écologie d'une rivière non aménagée des Alpes du Sud : Le Buëch (France) I - Evolution longitudinale des descripteurs physiques et chimiques
}

\author{
F. Vespinil \\ P. Légier ${ }^{2}$ \\ A. Champeau 1
}

Mots clés : Hydrochimie, eutrophisation, eutrophication, rivière, Alpes, analyse en composantes principales.

L'état hydrochimique du Buëch est appréhendé à partir d'une analyse multivariée de l'évolution longitudinale de 13 descripteurs du milieu aqueux. 31 points d'échantillonnage ont été choisis sur le cours d'eau axial et ses affluents.

De l'amont vers l'aval, on observe une augmentation progressive du degré de minéralisation des eaux. Ce continuum chimique est perturbé par des déversements d'eaux usées domestiques. L'analyse permet de distinguer deux phènomènes qui se manifestent de façon analogue : l'eutrophisation et l'eutrophication (ou pollution organique).

Ecology of a free-flowing river in the south of the Alps : the Buèch (France).

I - Longitudinal evolution of physical and chemical descriptors

Keywords : Hydrochemistry, natural eutrophication, man-made eutrophication, rivcr, Alps, principal componcnt analysis.

The hydrochemical composition of the Buëch river is studied through a multivariate data analysis of longitudinal evolution of thirteen descriptors of the water environment. Thirty-one sampling points on the river's course and its tributa. ries were chosen.

Upstream to downstream, there is a progressive increase in the water's degree of mineralization. These chemical continuum is disturbed by domestic waste water dumping. The analysis distinguishes natural eutrophication from man-made eutrophication.

\section{Introduction}

Une séric de trois publications devrait permettre de réaliser la monographie d'un des derniers cours d'eau subalpins non encore modifié par des aména. gements hydroélectriques. La première traite de l'évolution plus ou moins naturelle du chimisme de la rivière; la seconde, des communautés vivantes (algues et invertébrés benthiques) ; la troisième, par

Travail réalisé avec la collaboration technique de MM. НАвА (J.C.) et Garnier (R.)

1. Laboratoire d'Hydrobiologie, Université d'Aix-Marseille I, Centre Saint-Charles, Place Victor Hugo, 13331 Marseille Cédex 3.

2. Laboratoire d'Ecologie Animale, Hydrobiologic, Université d'Aix-Marseille III, Centre Saint-Jérôme, Rue Henri Poincaré, 13397 Marseille Cédex 4. un changement d'échelle de perception, envisage l'analyse des processus intervenant au niveau d'une perturbation d'origine organique.

Le but de cette présente étude est de mettre en évidence :

- d'une part l'évolution longitudinale de la charge ionique du cours d'eau en relation avec la nature des apports par ses affluents,

- d'autre part de révéler l'existence de discontinuités liées au transport d'altéragènes par certains affluents.

L'étude est menée dans deux situations hydrologiques différentes : la première se situe à la fin de la période des hautes eaux de la rivière (juin); la seconde, à la fin de la période d'étiage estival (septembre). 


\section{Le Buëch}

\subsection{Le cadre géographique}

Le Buëch reste, pour les Alpes du Sud, un des derniers exemples de rivière non aménagée. Son bassin versant de $1480 \mathrm{~km}^{2}$ couvre une partie du département de la Drôme et la majeure partie de celui des Hautes-Alpes. Il conflue avec la Durance au niveau de Sisteron dans les Alpes de Haute Provence.

Il est issu d'une multitude de petits torrents prenant naissance dans le Dévoluy à une alıitude de $2000 \mathrm{~m}$ environ (fig. 1). Son secteur amont de "type alpin " présente une pente de $10 \%$ (fig. 2). Il se forge un passage difficile de direction NE-SO dans le ravin des Clausis, jusqu'au village de Lusla-Croix-Haute ( $980 \mathrm{~m}$ d'altitude). A ce niveau, il rencontre deux ruisseaux: le Riou Froid (A1) et le Lunel (A2). Il emprunte ensuite une direction N-S en s'inserrant entre les montagnes de Mallemort (St-Julien en Beauchène: $920 \mathrm{~m}$, la Faurie : $840 \mathrm{~m}$ ) où il capte une série de petits cours d'eau dont les plus importants sont les Agnielles (A5) en rive gauche et l'Aiguebelle (A3) en rive droite; sa pente est alors de $3 \%$ et son lit mineur atteint déjà 4 à $5 \mathrm{~m}$ de large.

Plus en aval, entre les villages de Aspres sur Buèch et d'Eyguians, sa pente diminue encore et attein! $0,75 \%$. La rivière reçoit les eaux du Peti1 Buëch (A7) (son affluent le plus important), du Channe (A10) et du Riou (A11) en rive gauche, du Chauranne (A6),

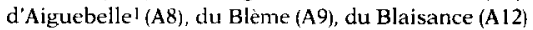
et du Céans (A13) en rive druite.

Entre Laragne et la confluence du Buëch avec la Durance au niveau de Sisteron ( $460 \mathrm{~m}$ d'altitude), la pente n'est plus que de $0,50 \%$ et la rivière s'étale considérablement; le lit est envahi par de nombreux îlots, quelquefois éphémères, qui donnent à l'écoulement un aspect de " tresses n. Dans ce secteur, le Buëch reçoit less saux du torrent de Méouge (A15) (deuxième affluent en importance) et du Clarescombes (A16) en rive droite, du Véragne (A14) qui véhi. cule les eaux usées de la ville de Laragne et de ses abattoirs en rive gauche.

1. Deux affluents portent le même nom, ils sont distingués dans l'étude par les sigles $\mathbf{A} 3$ et $\mathbf{A 8}$.

\subsection{Le régime hydrologique}

Le régime du Buëch esst de type nivopluvial. Il est caractérisé par 2 maxima très différents en amplitude. Les moyennes mensuelles établies pour la période 1906-1978 à la station Chambons (en amont de Serres) montrent que le 1er maximum qui se situe au printemps est très marqué (fig. 3); il est dû à l'action conjointe de la fonte des neiges et des précipitations. Le second, de plus faible amplitude correspond aux pluies automnales.

Dans ce secteur comme dans celui de Laragne (fig. 4) la période des hautes eaux du cycle hydrologique débute en février et se poursuit jusqu'à la fin du mois de mai. Le volume des eaux écoulées est en moyenne de $41 \mathrm{~m}^{3 /} / \mathrm{s}$ à Laragne, en 1983 mais on peut constater des pointes journalières de l'ordre de $328 \mathrm{~m}^{3 / \mathrm{s}}$ : le $16 \mathrm{mai}$, par exemple.

La période des basses eaux est relativement longue : 2,5 mois en moyenne et sévère, avec un débit de l'ordre de $3 \mathrm{~m}^{3 /} \mathrm{s}$. Comparée à ces moyennes établies sur 72 ans, l'année 1983 se révèle exceptionnelle. Ellc est caractérisée par un ćtiage estival très marqué : $2,05 \mathrm{~m}^{3 /} / \mathrm{s}$ en août contre une moyenne de $3.1 \mathrm{~m}^{3} / \mathrm{s}$ pour le même mois. De plus, la période des basses eaux qui commence tôt se poursuit jusqu'au début de l'hiver.

D'une manière générale le bassin versant subit l'influence montagnarde qui, par la fonte des neiges apporte une contribution importante au maximum de printemps. L'influence des pluies (en particulier des pluies automnales) sur le régime du cours d'eau est faible. L'influence méditerranéenne à laquelle est soumise la partie sud du bassin versant (Laragne) s'affirme nettement par la sècheresse estivale.

\subsection{Les activités économiques}

Les actjvités économiques dans la vallée appartiennent essentiellement au secteur primaire et consistent en cultures maraichères, céréalières, fourragères et en l'exploitation de grands vergers. L'élevage de bovins, d'ovins el de porcs s'étend de plus en plus ; corrélativement, les coopératives laitières et les abattoirs tendent à se développer.

Le secteur secondaire est moins bien représenté. On relève cependant, le long de la rivière, quelques 


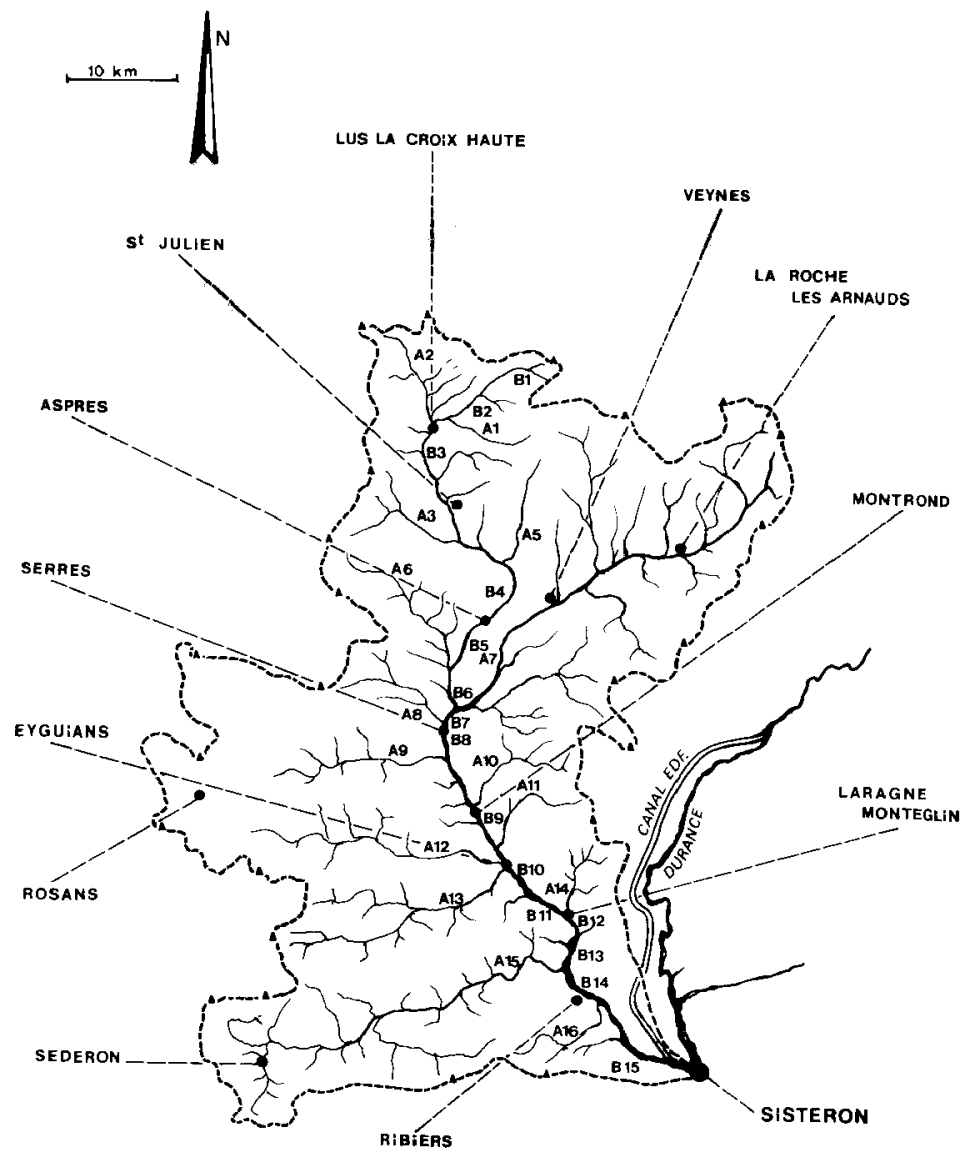

Fig. 1 : Le Buëch et son bassin versant. Localisation des stations (B1 à $\mathrm{B} 15$ = stations prospectées sur le cours principal. (Al à A16 = stations prospectées sur les affluents. 


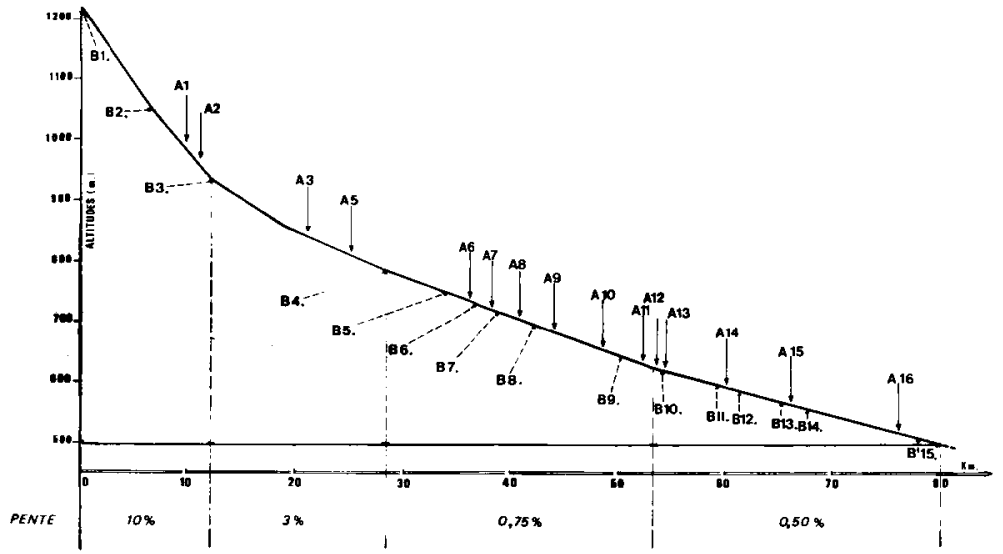

Fig. 2 : Profil en long du Buëch et situation des stations de prélèvement.

gravières permanentes ou occasionnelles dont le rôle sur la qualité de l'eau n'est pas à négliger.

Pour le secteur tertiaire enfin, les activités touristiques sont notables (campings, gítes ruraux, ...) en particulier dans la partie moyenne de la rivière.

\section{Résultats}

31 stations de mesure réparties sur le Buëch et ses affluents ont été prospectées au cours de 2 campagnes de prélèvements réalisées en juin et septembre 1983: 15 d'entre elles ont été prises sur le cours principal et 16 sur les affluents.

\subsection{Principales caractéristiques physiques et chimiques du cours d'eau axial}

13 composantes physiques et chimiques intervenant dans la détermination de la qualité des eaux, ont été relevées ou analysées. Ce sont : la conductivité, les duretés calcique et totale, l'alcalinité, les matières en suspension, l'oxydabilité au $\mathrm{KMnO}_{4}$ à chaud, les concentrations en ammoniaque, nitrites, nitrates, phosphates et chlorures, le pH et le pourcentage de saturation en oxygène dissous (Tableaux I et II).

\subsubsection{Minéralisation globale}

A l'image de la plupart des rivières alpines, la conductivité de la partie supérieure du cours d'eau est modérée. Elle était de $90 \mu \mathrm{S} / \mathrm{cm}$ en juin (Classe 3 dans l'échelle d'interprétation proposée par Nisbet \& Vemeaux, 1970), tandis que la partie inférieure présentait une minéralisation forte à très forte avec $413 \mu \mathrm{S} / \mathrm{cm}$ au niveau de la confluence du Buëch et de la Durance (classe 7 des mêmes auteurs 1 ). En septembre, les valeurs étaient de $193 \mu \mathrm{S} / \mathrm{cm}$ (classe 4) à la station B1 et de $422 \mu \mathrm{S} / \mathrm{cm}$ (classe 7) en B15. Comme cela a été observé ailleurs (Giudicelli \& al. 1980; Doledec 1986), la

1. Dans la suite du texte, l'attribution de classes des facteurs abiotiques se fera par référence aux valeurs repères proposées par Nisbet \& Verneaux $(1970)$. 


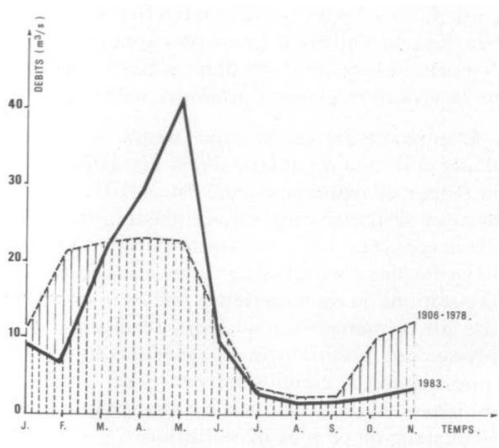

Fig. 3 : Evolution mensuelle des débits jaugés aux Cham. bons (courbes en tirés: moyenne effectuée sur la période 1906-1978; courbe en trait plein: évolution pour 1983). (Les valeurs manquent pour décembre).

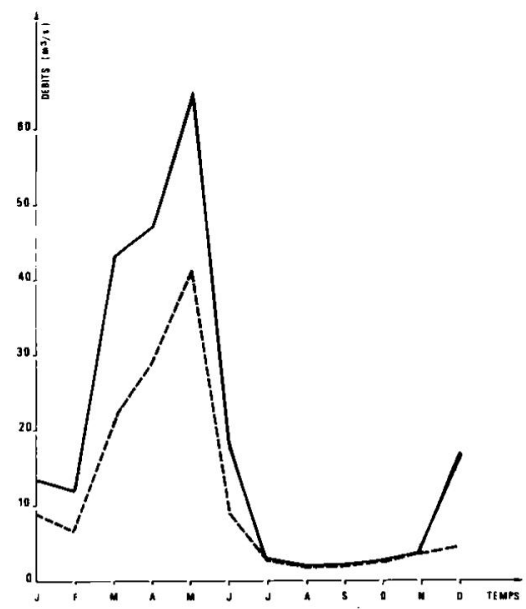

Fig. 4 : Evolution mensuelle des débits jaugés en 1983 dans le secteur supérieur du Buëch (Chambons : courbes en tirés) et dans le secteur inférieur (Laragne : courbe en trait plein). conductivité croît progressivement de l'amont vers l'aval. Ici, l'augmentation générale du degré de minéralisation est liée d'une part, à l'augmentation de la superficie du bassin versant et du débit et donc aux apports croissants de minéraux solubles et, d'autre part, à une augmentation du taux de pollution vers l'aval du Buëch.

Les teneurs en $\mathrm{CaCO}_{3}$ sont celles d'une eau dure : elles sont respectivement égales à $90 \mathrm{mg} / \mathrm{l}$ et $150 \mathrm{mg} / \mathrm{l}$ en juin et septembre en amont (classes 5 et 6) et à $195 \mathrm{mg} / \mathrm{l}$ et $215 \mathrm{mg} / \mathrm{l}$ en aval (classe 7). Les concentrations calciques étant très dominantes par rapport aux concentrations magnésiennes, elles impriment à la dureté totale son évolution longitudinale. Comme pour la conductivité, les valeurs mesurées sont plus élevées en septembre qu'en juin et les écarts observés plus réduits.

Les teneurs en calcium sont essentiellement tributaires de la nature des terrains traversés ; elles sont élevées dès la source : 75 et $120 \mathrm{mg} \mathrm{de} \mathrm{CaCO}_{3} / 1$ (résultats des analyses effectuées respectivement en juin et en septembre). Elles augmentent de façon globale tout le long du cours d'eau pour atteindre les valeurs de $165 \mathrm{mg} / \mathrm{l}$ et $155 \mathrm{mg} / \mathrm{l}$ aux deux périodes d'étude en B15.

Les teneurs en magnésium des eaux du Buëch sont importantes, elles varient entre 10 et $55 \mathrm{mg} / \mathrm{l}$ (classes 4,5 et 6 ). Cette dureté magnésienne est due en grande partie à la présence de calcaires dolomitiques ou de dolomies.

Les pH relevés varient entre 7,8 et 8,2 et traduisent des eaux faiblement ou moyennement alcalines (classes 5 et 6).

Les concentrations en $\mathrm{HCO}_{3}-$ varient entre $90 \mathrm{mg} / \mathrm{l}$ en $\mathrm{B} 1$ et $165 \mathrm{mg} / \mathrm{l}$ en B15 en juin, entre $120 \mathrm{mg} / \mathrm{l}$ et $180 \mathrm{mg} / \mathrm{l}$ aux mèmes stations en septembre (classes 3, 4 puis 4 et 5 ). Ces valeurs traduisent une alcalinité moyenne et se rapportent à des eaux très productives possédant un pouvoir tampon très élevé. Les variations de l'alcalinité sont à rapprocher de celles de la conductivité et de la teneur en alcalino-terreux et suivent la mème évolution globale le long du cours d'eau.

Jusqu'à la station B4, les teneurs en chlorures relevées sur le Buëch, n'excèdent pas $10 \mathrm{mg} / \mathrm{l}$, ce qui est généralement le cas des zones supérieures des réseaux hydrographiques. Cependant, à partir de cette station, elles augmentent régulièrement et 
atteignent $30 \mathrm{mg} / \mathrm{l}$ à la station $\mathrm{B} 15$ en juin et $35 \mathrm{mg} / \mathrm{l}$ en septembre (classe 5); elles témoignent alors d'une légère pollution du milieu.

\subsubsection{Matières en suspension}

L'estimation des matières en suspension permet de déterminer la quantité de matières insolubles aussi bien d'origine minérale (pouvoir d'érosion du cours d'eau ou présence de gravières) qu'organique (déversement d'effluents domestiques). Ces teneurs sont liées aux événements hydrobiologiques de la rivière. Dans le cours principal du Buëch, elles sont toujours inférieures à $25 \mathrm{mg} / 1$ (classes 1 et 2); dans un seul affluent : le Riou (code Al1) elles ont atteint $52,2 \mathrm{mg} / \mathrm{l}$ en septembre.

\subsubsection{Oxydabilité}

Pour les eaux du Buëch, l'oxydation a été réalisée à chaud en milieu alcalin. Les valeurs obtenues oscillent entre 0,45 et $0,72 \mathrm{mg} / \mathrm{l}$ en juin, entre 0,35 et $1,17 \mathrm{mg} / \mathrm{l}$ en septembre, valeurs habituellement observées dans des eaux courantes normales. Quelle que soit la période de mesure, les pourcentages en oxygène dissous sont toujours égaux ou supérieurs à 100 (classe 1); ils caractérisent des eaux de bonne qualité biologique.

\subsubsection{Composés azotés et phosphorés}

Les valeurs de nitrates relevées oscillent entre $0,94 \mathrm{mg} / \mathrm{l}$ à la station $\mathrm{B} 1$ (classe 1) et $2,18 \mathrm{mg} / \mathrm{l}$ à la station B15 (classe 2) en juin. On constate dans l'ensemble, une augmentation régulière au fur et à mesure que croît la distance à la source et corréla. tivement avec le degré d'eutrophie dû aux apports latéraux et à l'accumulation des matières organiques endogènes. Une teneur relativement forte $(1,56 \mathrm{mg} / 1)$ est relevée en $\mathrm{B} 1$ en septembre. Ce phénomène a déjà été souligné pour d'autres rivières (Martin 1979).

Les nitrites proviennent généralement des transformations intermédiaires de l'ammoniaque en nitrates : leur présence constitue donc le plus sou. vent, l'indice d'une pollution organique en évolution. Dans ses secteurs supérieurs et moyens (jusqu'à Serres), le Buëch ne recèle que des traces de nitrites : les valeurs observées plus en aval sont de l'ordre du centième de $\mathrm{mg} / \mathrm{l}$ (classe 1). ce qui traduit des eaux pures ou à autoépuration active. En Septembre, les concent rations mesurées sont un peu plus importantes, notamment dans la partie inférieure de la rivière et peuvent atteindre $0,03 \mathrm{mg} / \mathrm{l}$.

L'ammoniaque est presque toujours le témoin d'une pollution organique. Il est décelable lorsque la teneur en eau en oxygène est insuffisante pour assurer sa transformation. Le phénomene ne s'observe généralement pas dans le Buëch, milieu bien oxygéné ; les concentrations mesurées dans certaines stations du cours moyen et inférieur sont de l'ordre du centième de $\mathrm{mg} / \mathrm{l}$, elles traduisent à leur niveau une pollution insidieuse sensible. L'azote ammoniacal a essentiellement pour origine les déversements domestiques, il constitue un excellent indicateur de ce type de pollution.

Notre étude ne porte que sur les orthophosphates qui constituent la forme de phosphore assimilable par les algues. Lorsque les concent rations dans les caux naturelles sont supérieures à 0,1 ou $0,2 \mathrm{mg} / \mathrm{l}$, elles traduisent une pollution par des eaux domestiques contenant des phosphates organiques et des détergents synthétiques, et (ou) par des eaux de ruissellement. La partie supérieure de l'axe principal du Buëch présente des valeurs inférieures à $10 \mu \mathrm{g} / \mathrm{l}$; les teneurs augmentent vers l'aval mais ne dépassent pas $0,02 \mathrm{mg} / \mathrm{l}$ (la valeur de $0,197 \mathrm{mg} / \mathrm{l}$ enre. gistrée à la station $\mathrm{B} 12$ en septembre paraît accidentelle).

En conclusion, les eaux du Buëch sont des eaux calcaires, dures, légèrement à moyennement alcalines, au pouvoir tampon élevé, très productives. On ne relève pas de zone critique de pollution au sens de Nisbet \& Verneaux (1970), mais les teneurs en nitrites, ammoniaque et phosphates traduisent un apport insidieux d'eaux "vannes" dans la partie inférieure du cours d'eau.

\section{Interprétation par l'A.C.P.}

Afin de révéler le rôle et l'importance des différents affluents sur le chimisme du cours d'eau axial, deux analyses en composants principales (A.C.P.) prenant en compte 12 des 13 variables précédemment citées, ont été réalisées séparérnent pour les mois de juin et de septembre. Elles portent sur les 31 stations retenues (le pourcentage de saturation en oxygène, toujours supérieur à 100 , n'a pas été 
considéré). Cette méthode d'analyse multidimensionnelle a déjà été employée pour aborder l'étude de la pollution de la Seine (Landreau 1974) et de la qualité des eaux de rivière (Guilbot 1981 ; Guilbot \& al. 1982).

Les figures 5 et 8 montrent la contribution qu'apportent les différents descripteurs physiques ou chimiques à la formation des axes principaux I et II, ainsi que la projection dans ce plan, des angles de corrélation entre descripteurs. Le cercle de corrélation (cercle unité) et le cercle de contribution équilibrée des descripteurs ont été ajoutés. Ce dernier permet de remarquer que la contribution de chacun des descripteurs à l'espace réduit est plus élevée que le voudrait l'hypothèse d'une contribution égale de leur part à tous les axes principaux. exception faite des $\mathrm{pH}$ relevés en juin. Dans la suite de notre raisonnement, nous ne tiendrons plus compte de cette variable pour le mois considéré.

\subsection{A.C.P. menée sur les échantillons prélevés en juin}

Deux ensembles de points s'individualisent sur la figure 5. L'examen de la matrice de corrélation entre variables révèle que le premier ensemble est constitué par des descripteurs très bien corrélés entre eux ; ce sont la conductivité, les duretés totale et calcique, l'alcalinité (état ionique) et les formes oxydées de l'azote : nitrites et nitrates. Ce premier ensemble traduit un enrichissement ionique progressif "naturel " des eaux du Buëch, lié pour l'essentiel à l'évolution de certains paramètres de l'amont vers l'aval tels que l'altitude, la pente, la surface du bassin versant ou le débit par exemple. Ces descripteurs physiques et chimiques contribuent fortement à la formation de l'axe I; en effet, leur corrélation avec la composante principale $C_{1}$ est importante : la part de variance expliquée par la composante principale $\mathrm{C}_{1}$ est égale à $39,06 \%$. Le premier axe principal décrit donc l'évolution générale du degré de minéralisation et corrélativement du degré d'eutrophie du cours d'eau.

Le second ensemble regroupe des descripteurs du milieu tels que la matière organique, les orthophosphates et la forme réduite de l'azote : l'ammoniaque, qui caractérisent essentiellement des apports d'eaux uscies domestiques. $C_{2}$ dont la part de variance expliquée est égale à $19,91 \%$, est fortement corrélée avec cet ensemble ; le second axe principal traduit donc l'intensité de la pollution apportée par des deversements urbains d'eaux usées.

L'évolution des conditions de milieu de l'amont vers l'aval traduite par l'axe I, est confirmée par la place de la station $\mathrm{Bl}$ en position très net tement positive sur l'axe et à l'opposé, par celle de la station $\mathrm{B} 15$ en position négative sur le même axe. Les autres stations étudiées sur le cours principal se répartissent à peu près entre ces deux points en fonction de leur distance à la source. En effet, si l'on porte en ordonnées, comme sur le graphique 6 , les coordonnées des stations calculées à partir de l'A.C.P. sur l'axe principal et en abscisse leur distance par rapport à la source, la courbe tracée croít progressivement au fur et à mesure que croît le degré de minéralisation du cours d'eau. Cette évolution normale se réalise pleinement entre la source du Buëch et la station B8, soit sur une distance de $50 \mathrm{~km}$ environ ; elle est directement sous la dépendance des apports d'eaux provenant des différents affluents. Dans la partie supérieure du réseau hydrographique, la composition chimique de ces derniers, proche de celle du cours principal, ne provoque pas de discontinuité notable dans le « continuum chimique » amont-aval. A partir de la station B8 apparaissent des perturbations : ainsi, la position nettement négative de la station B9 sur la figure 5 s'explique par l'arrivée dans le Buëch, des eaux très minéralisées du Channe (A10) qui apportent en grande quantité des nitrates au cours principal. La position de la station B12, à gauche du point B15 ( $\mathrm{fig}$. 5), en projection orthogonale sur l'axe I, est due à l'arrivée de rejets domestiques véhiculés par les eaux du Véragne (A14). Ce type de perturbation est caractérisé essentiellement par l'apport d'orthophosphates, d'ammoniaque et par un accroissement de l'oxydabilité, descripteurs qui caractérisent l'axe II. Leur contribution sur l'axe I n'est cependant pas à négliger. De plus, la concentration d'autres éléments comme l'alcalinité, les nitrates, les chlorures et donc la conductivité (variables qui présentent une forte contribution sur l'axe I), augmente également dans les effluents urbains ; la conséquence graphique est un glissement vers la gauche de l'axe I du point représentatif de la station qui subit les effets de ces déversements.

D'autres affluents comme la Méouge (A15) par exemple, véhiculent des eaux moins minéralisées 


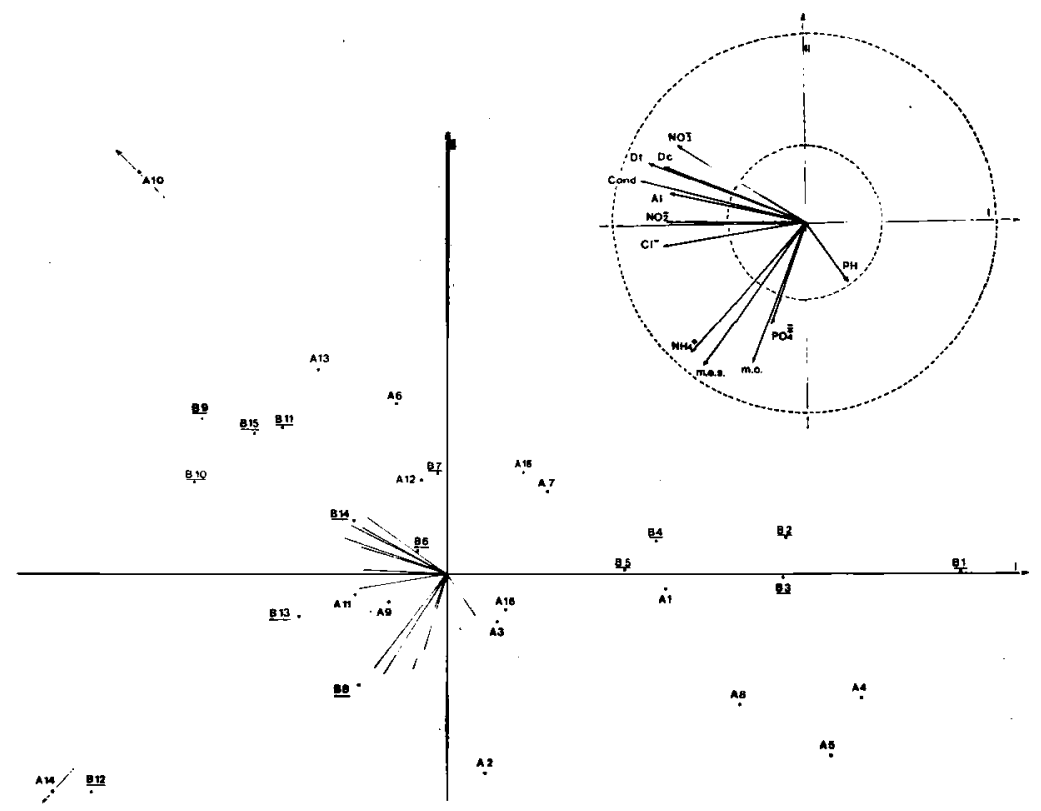

Fig. 5 : Représentation dans le plan I-II des descripteurs du milieu en juin et projection des points stations.

(Légendes $: \mathrm{Dc}$. = Dureté calcique, $\mathrm{Dt}$. = Duretê totale, Cond. = Conductivité, Al. = Alcalinité, m.e.s. $=$ Matières en suspension, m.o. = Matière organique).

que celles du Buëch, ce qui provoque au contraire, un déplacement vers la droite de la station B14, située en aval.

Le second axe principal qui reflète la contribution des apports exogenes polluants dans l'hydrochimie du Buëch, individualise deux zones (fig. 7) : la première regroupe les stations du secteur amont de la rivière, elle est caractérisée par une bonne qualité des eaux et par une relative constance des caracté. ristiques hydrochimiques; la seconde, située en aval de la station B6, est soumise à des fluctuations plus importantes. Dans ce deuxième groupements de stations, on remarque notamment la présence du point Al4 en coordonnée très négative sur l'axe (fig. 5); cet affluent reçoit les eaux usées de la ville de Laragne, son impact sur le Buëch est nettement présent au niveau de la station B12 (fig. 5 et 7) située à $300 \mathrm{~m}$ en aval de la confluence des deux cours d'eau; la perturbation occasionnée est encore sensible à la station B13. Les points représentant les stations B12 et B 13 sont de ce fait situés dans le quadrant négatif du plan défini par les axes I et II.

La station $\mathrm{B} 8$ se trouve en position négative sur l'axe II (fig. 5). Cet axe individualise donc les deux stations situées immédiatement en aval des deux plus grandes villes riveraines: Serres pour B8 et Laragne pour B12 (fig. 7). La perturbation apportee par la ville de Laragne, plus importante en intensité 


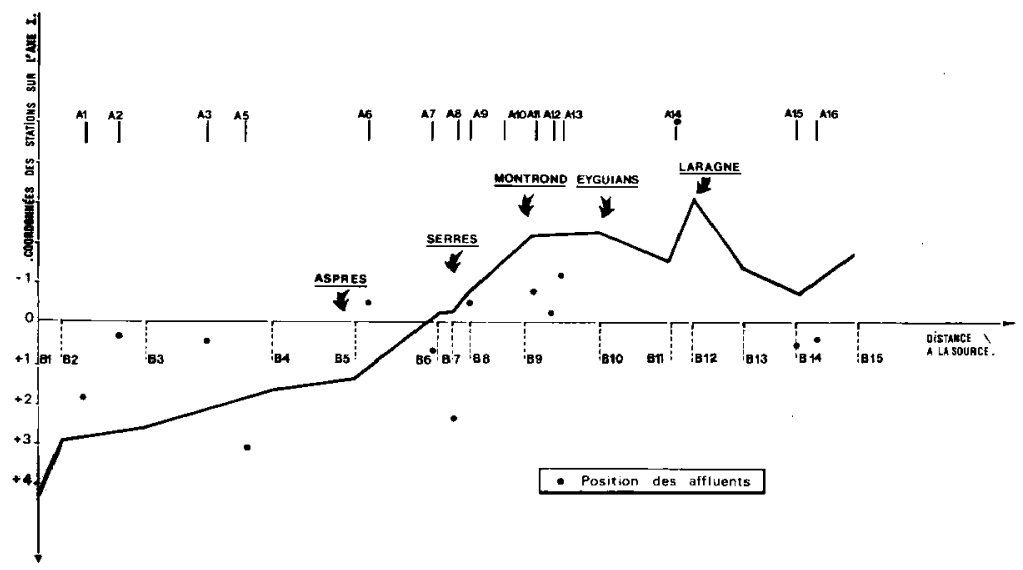

Fig. 6 : Evolution de l'amont vers l'aval de la minéralisation des eaux du Buëch en juin, codée par les coordonnées des stations sur le premier axe d'une A.C.P. portant sur les paramètres chimiques.

ponctuelle et dont les répercussions se font sentir sur une plus grande portion de cours d'eau, fera ultérieurement l'objet d'une étude approfondie.

\subsection{A.C.P. menée sur les échantillons prélevés en septembre}

La part de variance expliquée par la composante principale $C_{1}$, est plus importante en septembre qu'en juin $(43,28 \%$ contre $39,06 \%)$; en revanche, elle est un peu plus faible en ce qui concerne la composante $C_{2}(18,09 \%$ au lieu de $19,91 \%)$.

La composante principale $C_{1}$ acquiert donc une signification supplémentaire entre ces deux périodes. En effet, les variables : conductivité, dureté, alcalinité et nitrates restent toujours étroitement corrélées entre elles, comme elles l'étaient en juin. Mais à la fin de la période d'étiage, elles sont aussi corrélées à la matière organique, ce qui témoigne non seulement d'une augmentation du degré de minéralisation mais également d'une eutrophisation accrue de l'ensemble du cours d'eau (fig. 8).
En septembre, seuls les orthophosphastes et la forme réduite de l'azote $\left(\mathrm{NH}_{4}{ }^{+}\right)$déterminent la composante $\mathrm{C}_{2}$ (les deux descripteurs sont d'ailleurs très fortement corrélés entre eux : 0,975 ). La composante $\mathrm{C}_{2}$ conserve la même signification qu'au mois de juin.

Cependant, la situation hydrochimique est moins claire au cours de la deuxième période : les paramètres traduisant une eutrophisation « naturelle " ou "provoquée " sont mieux corrélés entre eux et influencent de façon plus importante les deux axes principaux.

Sur la figure 8, les stations occupent sensiblement les mêmes positions sur le premier axe principal au cours des deux campagnes d'analyses. Sur le second axe apparaissent à nouveau les apports du Véragne (A14) et leur impact sur la station B12. Cependant, trois caractères déterminent le positionnement des stations dans le plan I-II :

- les stations du secteur supérieur de la rivière jusqu'au point B4 sortent en opposition avec les facteurs dureté calcique, dureté totale, alcalinité, 


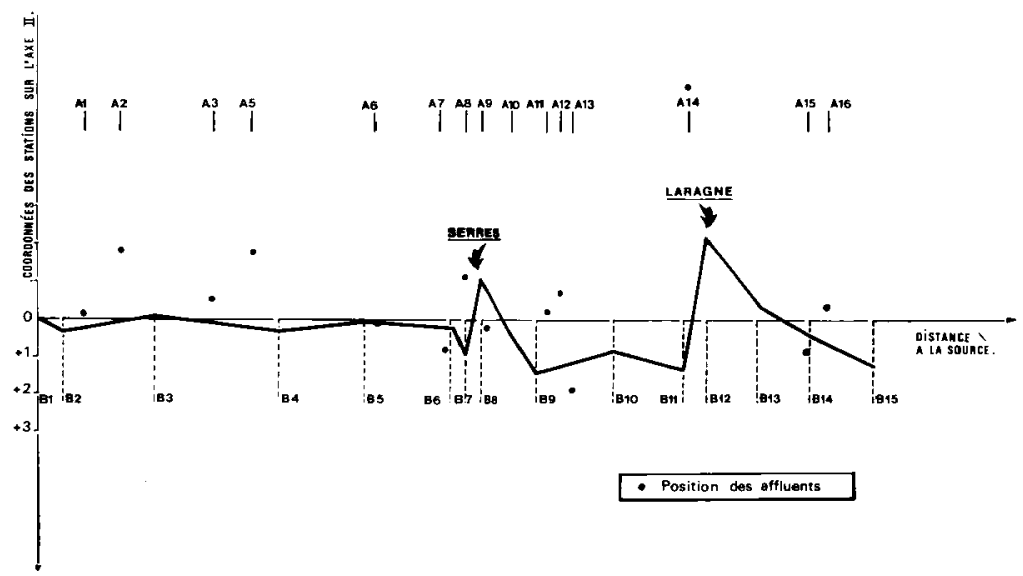

Fig. 7 : Evolution du degré de pollution des eaux du Buëch en juin, codée par les coordonnées des stations sur le deuxième axe d'une A.C.P. portant sur les paramètres chimiques.

conductivité, matière organique, ... qui déterminent l'axe I.

- les stations A14 et B 12 sortent dans le sens des plus fortes valeurs des descripteurs de pollution $\mathrm{NH}_{4}+$ et $\mathrm{PO}_{4}{ }^{3-}$.

les stations aval sortent du côté négatif de l'axe II, dans le sens des plus fortes valeurs de minéralisation et d'eutrophisation naturelle.

Cette répartition des points " stations " s'effectue donc plus en fonction de secteurs de plan qu'en fonction des axes, elle est due au grand nombre de facteurs qui déterminent l'axe I; certains d'entre eux sont très étroitement corrélés; citons : l'alcalinité, la dureté calcique et la dureté totale dont les corrélations sont comprises entre 0,83 et $0,89 \%$. Ces facteurs ont sensiblement la même évolution et participent fortement à la détermination des coordonnées des stations dans le plan factoriel I-II.

Toutefois, la courbe présentée par la figure 9 traduit encore en septembre l'évolution de la minéra. lisation et du degré d'eutrophie des eaux du Buëch. Elle possède sensiblement la même allure que celle de la figure 6 pour le mois de juin. De la source jusqu'au niveau de la station B8, on observe peu de différence d'une période d'analyse à l'autre, seule la minéralisation des eaux de la station $\mathrm{B} 5$ change légèrement. En aval de cette station, les conditions abiotiques relevées en $\mathrm{B} 9$ et $\mathrm{B} 10$ sont à l'inverse, très nettement différentes aux deux époques : la minéralisation des eaux, importante en juin, est paradoxalement beaucoup plus faible en septembre. En juin, l'apport massif de nit rates par le Channe (A10) au niveau de la station $B$ 9, ainsi que les concentrations élevées de chlorures enregistrées en $B 10$ sont à l'origine de la situation très positive sur le plan factoriel I-II des deux points-stations (dans le sens d'une plus grande minéralisation et d'une plus grande eutrophisation des eaux). En septembre, par suite de la cessation de ces apports accidentels, les deux points-stations se rapprochent de l'origine des axes. Par contre, le Riou (A11), autre affluent, est très fortement minéralisé en septembre, il participe au retour des eaux du Buëch à une situation semblable à celle observée en juin, à partir de la station B11. 


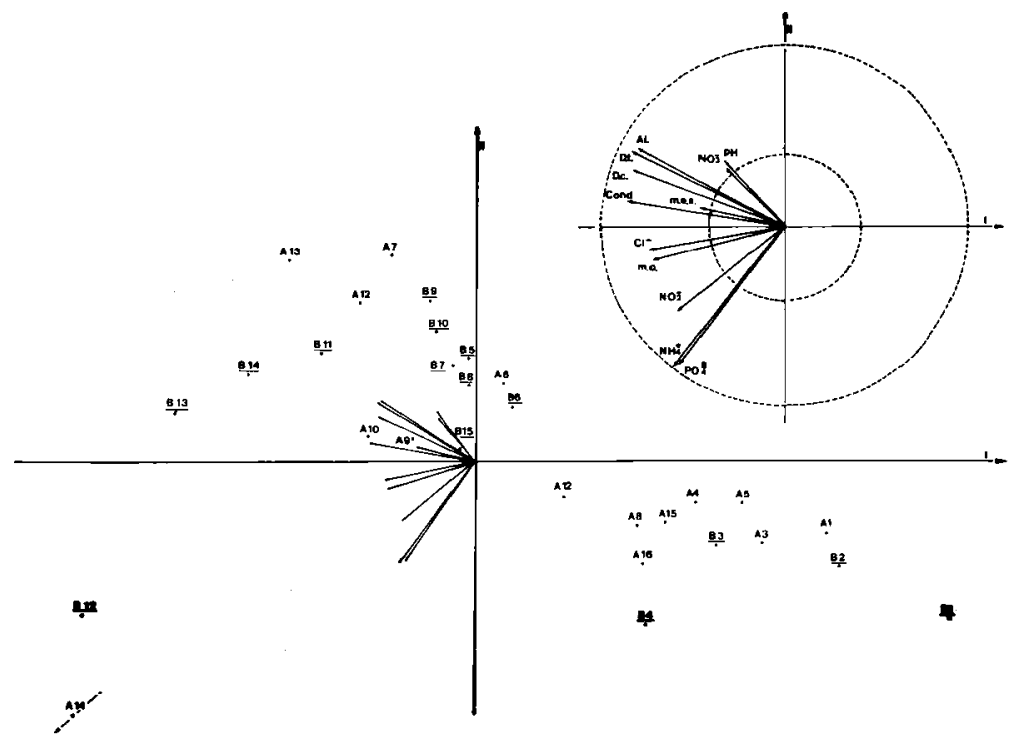

Fig. 8 : Représentation dans le plan I-II des descripteurs du milieu en septembre et projection des points-stations. (Légendes $: \mathbf{D c}=$ Dureté calcique, $\mathbf{D t}$. = Dureté totale, Cond. = Conductivité, Al. = Alcalinité, m.e.s. = Matières en suspension, m.o. = Matière organique).

\section{Conclusion}

A la suite de cette étude menée à l'échelle du réseau hydrographique, il apparaît donc que le chimisme du Buëch subit une évolution naturelle de ses composantes de l'amont vers l'aval. A cette évolu. tion " normale " se superpose l'influence d'un certain nombre d'impacts liés aux activités économiques riveraines.

Ainsi, au moment des prélèvements de juin, les pratiques agricoles sont probablement à l'origine de modifications patentes mais plus ou moins accidentelles, survenues dans la composition des eaux du cours principal de la rivière, ent re les agglomérations de Montrond et d'Eyguians (B9, B10).

En revanche, les déversements d'eaux usées de la ville de Laragne et de ses abattoirs, par le canal que représente le Véragne (A14), sont à l'origine de modifications chroniques du chimisme du Buëch.

En fin de compte (et si l'on se réfère à Dajoz, 1977), il est permis, semble-t-il, de conclure que l'A.C.P. menée sur le chimisme des eaux du Buëch et de ses affluents traduit, par l'axe I, une évolution naturelle correspondant à l'augmentation progressive du niveau trophique des eaux de l'amont vers l'aval de la rivière : augmentation imputable à des phénomènes * naturels " tels que le lessivage des terrains traversés ou la concentration des eaux en sels à la suite d'une période de sècheresse prolongée. Il s'agit donc du phénomène d'eutrophisation.

L'axe II traduit, pour sa part, un accroissement anthropique de la minéralisation des eaux sur un tronçon particulier de la rivière. Cet accroissement ne peut être constaté que par référence aux 
Tableau I : Résultats des analyses effectuées sur l'ensemble du Buëch et ses affluents en juin. (Légendes : Cond. = conductivité, $\mathrm{Dc}=$ Dureté calcique, $\mathbf{D t}=$ Dureté totale, $\mathbf{A l}$. = Alcalinité, $\mathrm{m} . \mathrm{e} . \mathrm{s}=$ Matières en suspension, m.o. $=$ Matière organique $\mathrm{t} r=$ traces).

\begin{tabular}{|c|c|c|c|c|c|c|c|c|c|c|c|c|}
\hline $\begin{array}{l}\text { Septem- } \\
\text { bre } \\
1984\end{array}$ & 픔 & 总焉 & 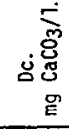 & 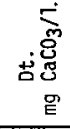 & 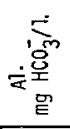 & $\stackrel{\dot{5}}{\circ}$ & $\begin{array}{l}\dot{\varphi} \\
\dot{E} \\
\dot{E}\end{array}$ & $\begin{array}{l}\dot{\Sigma} \\
\dot{\circ} \\
\dot{E} \mathscr{E}\end{array}$ & 울 핱 & '官 & +्ञ̄ं & 'ं \\
\hline B1 & 7,8 & 193 & 120 & 150 & 120 & 7,5 & 3,4 & 0,37 & tr. & 1,56 & 0,00 & 0,01 \\
\hline B2 & 7,8 & 232 & 140 & 160 & 140 & 10,0 & 5,4 & 0,35 & $t r$. & 1,10 & 0,00 & 0,01 \\
\hline A1 & 7,9 & 213 & 145 & 160 & 140 & 7,5 & 3,8 & 0,50 & tr. & 0,88 & 0,00 & 0,01 \\
\hline$A 2$ & 8,0 & 242 & 160 & 180 & 175 & 10,0 & 13,0 & 0,62 & $\rho, 02$ & 1,51 & 0,00 & 0,03 \\
\hline B3 & 7,8 & 258 & 145 & 185 & 145 & 10,0 & 6,4 & 0,42 & 0,01 & 1,51 & 0,00 & 0,01 \\
\hline A3 & 7,7 & 265 & 140 & 170 & 170 & 12,5 & 4,8 & 0,40 & tr. & 0,55 & 0,00 & 0,01 \\
\hline A4 & 7,8 & 283 & 155 & 180 & 165 & 10,0 & 4,0 & 0,45 & tr. & 0,90 & 0,02 & 0,01 \\
\hline A5 & 7,9 & 261 & 150 & 180 & 160 & 12,5 & 1,4 & 0,37 & tr. & 0,24 & 0,00 & 0,01 \\
\hline B4 & 7,8 & 287 & 150 & 170 & 155 & 15,0 & 13,0 & 0,42 & 0,03 & 0,62 & 0,01 & 0,01 \\
\hline B5 & 7,8 & 345 & 180 & 200 & 200 & 10,0 & 2,2 & 0,22 & 0,01 & 2,48 & 0,02 & 0,01 \\
\hline$A 6$ & 7,9 & 392 & 150 & 185 & 170 & 25,0 & 9,4 & 0,85 & $\operatorname{tr}$ & 2,92 & 0,00 & 0,01 \\
\hline B6 & 7,7 & 423 & 170 & 200 & 175 & 15,0 & 1,8 & 0,47 & tr. & 2,06 & 0,03 & 0,01 \\
\hline A7 & 8,0 & 421 & 170 & 240 & 180 & 25,0 & 1,8 & 0,35 & $\operatorname{tr}$ & 2,70 & 0,00 & 0,01 \\
\hline B7 & 8,0 & 382 & 175 & 200 & 180 & 20,0 & 1,2 & 0,30 & 0,01 & 2,30 & 0,02 & 0,01 \\
\hline A8 & 8,1 & 317 & 140 & 185 & 155 & 17,5 & 5,8 & 0,55 & 0,01 & 0,34 & 0,00 & 0,03 \\
\hline B8 & 8,0 & 318 & 170 & 205 & 180 & 17,5 & 5,0 & 0,60 & 0,01 & 2,13 & 0,02 & 0,02 \\
\hline A9 & 7,8 & 341 & 175 & 195 & 190 & 10,0 & 45,0 & 1,67 & tr. & 0,33 & 0,03 & 0,01 \\
\hline A10 & 8,1 & 395 & 140 & 180 & 170 & 32,5 & 49,0 & 1,07 & 0,01 & 2,32 & 0,05 & 0,01 \\
\hline$B 9$ & 8,1 & 374 & 175 & 200 & 180 & 30,0 & 7,0 & 0,42 & $\operatorname{tr}$ & 2,60 & 0,02 & 0,01 \\
\hline Al1 & 8,0 & 420 & 230 & 285 & 220 & 25,0 & 52,2 & 2,40 & 0,01 & 1,26 & 0,04 & 0,01 \\
\hline A12 & 8,1 & 339 & 180 & 220 & 190 & 15,0 & 9,6 & 0,82 & 0,01 & 2,48 & 0,00 & 0,01 \\
\hline A13 & 8,1 & 369 & 200 & 220 & 205 & 15,0 & 9,0 & 1,52 & $\mathrm{tr}$ & 1,47 & 0,00 & 0,01 \\
\hline$B 10$ & 8,1 & 388 & 170 & 210 & 175 & 35,0 & 3,2 & 0,52 & tr. & 1,72 & 0,00 & 0,01 \\
\hline B11 & 7,9 & 453 & 160 & 210 & 190 & 55,0 & 5,4 & 0,35 & 0,01 & 2,68 & 0,00 & 0,01 \\
\hline A14 & 7,9 & 482 & 190 & 210 & 185 & 55,0 & 13,2 & 2,15 & 0,04 & 1,52 & 0,50 & 0,56 \\
\hline$B 12$ & 7,9 & 430 & 180 & 230 & 180 & 55,0 & 11,4 & 1,17 & 0,03 & 1,96 & 0,09 & 0,20 \\
\hline 813 & 8,0 & 446 & 180 & 220 & 190 & 57,5 & 13,0 & 0,75 & 0,03 & 1,77 & 0,02 & 0,01 \\
\hline A15 & 8,2 & 290 & 140 & 170 & 145 & 15,0 & 4,0 & 0,77 & 0.01 & 0,94 & 0,00 & 0,01 \\
\hline B14 & 8,0 & 436 & 180 & 235 & 185 & 45,0 & 8,8 & 0,67 & 0,02 & 1,36 & 0,00 & 0,01 \\
\hline A16 & 7,9 & 329 & 130 & 185 & 150 & 20,0 & 4,2 & 0,90 & 0,01 & 1,01 & 0,00 & 0,01 \\
\hline B15 & 7,8 & 422 & 155 & 215 & 180 & 35,0 & 10,0 & 0,37 & 0,01 & 0,66 & 0,00 & 0,01 \\
\hline
\end{tabular}


Tableau II : Résultats des analyses effectuèes sur l'ensemble du Buëch et ses affluents en septembre. (Légendes : Cond. = conductivité, $\mathrm{Dc} .=$ Dureté calcique, $\mathrm{Dt}=$ Dureté totale, $\mathrm{Al}$. = Alcalinité, m.e.s. = Matières en suspension, m.o. $=$ Matière organique, $\mathbf{t r}=\mathbf{t r a c e s}$ ).

\begin{tabular}{|c|c|c|c|c|c|c|c|c|c|c|c|c|}
\hline $\begin{array}{l}\text { Juin } \\
1984\end{array}$ & 吉 & 总 $\frac{E}{\delta}$ & ப. & 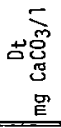 & 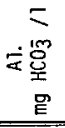 & 宁 $\overline{\bar{E}}$ & $\stackrel{\dot{y}}{\dot{E}} \overline{\text { Dे }}$ & 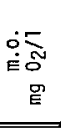 & 呈 & 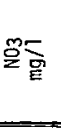 & 㐭品 & ${ }^{\prime} \overline{8}$ \\
\hline 81 & 7,8 & 59 & 75 & 90 & 90 & 5,0 & 1,2 & 0,45 & tr. & 0,94 & 0,00 & 0,01 \\
\hline 82 & 7,8 & 86 & 115 & 135 & 115 & 7,5 & 1,4 & 0,47 & $\mathrm{tr}$ & 1,06 & 0,00 & 0,03 \\
\hline $\mathrm{Al}$ & 7,8 & 132 & 145 & 155 & 135 & 10,0 & 1,0 & 0,60 & $\operatorname{tr}$ & 0,86 & 0,01 & 0,06 \\
\hline A2 & 7,9 & 182 & 160 & 180 & 155 & 5,0 & 3,6 & 0,45 & $\operatorname{tr}$ & 1,44 & 0,03 & 0,23 \\
\hline B3 & 8,1 & 152 & 125 & 150 & 135 & 5,0 & 2,0 & 0,50 & tr & 0,90 & 0,00 & 0,01 \\
\hline $\mathrm{A} 3$ & 7,9 & 205 & 185 & 195 & 165 & 7,5 & 3,2 & 0,6 & tr. & 0,42 & 0,03 & 0,06 \\
\hline A4 & 8,0 & 183 & 40 & 65 & 140 & 12,5 & 2,4 & 0,52 & tr. & 0,54 & 0,01 & 0,01 \\
\hline A5 & 8,2 & 176 & 60 & 100 & 160 & 7,5 & 1,8 & 0,67 & tr. & 0,22 & 0,00 & 0,01 \\
\hline B4 & 8,0 & 188 & 140 & 165 & 150 & 12,5 & 0,8 & 0,55 & tr. & 0,76 & 0,00 & 0,01 \\
\hline B5 & 8,1 & 211 & 145 & 175 & 150 & 12,5 & 2,0 & 0,47 & tr. & 0,76 & 0,02 & 0,01 \\
\hline$A 6$ & 7,8 & 324 & 175 & 210 & 175 & 10,0 & 2,2 & 0,60 & $\mathrm{tr}$ & 2,42 & 0,00 & 0,02 \\
\hline B6 & 8,0 & 251 & 195 & 235 & 155 & 10,0 & 1,6 & 0,65 & $\mathrm{tr}$ & 1,62 & 0,04 & 0,01 \\
\hline A7 & 8,0 & 255 & 145 & 190 & 135 & 17,5 & 3,8 & 0,42 & tr. & 1,96 & 0,00 & 0,02 \\
\hline B7 & 7,8 & 341 & 155 & 180 & 165 & 12,5 & 2,0 & 0,60 & tr. & 2,30 & 0,02 & 0,01 \\
\hline$A 8$ & 8,0 & 327 & 55 & 90 & 155 & 10,0 & 3,6 & 0,62 & tr. & 0,44 & 0,01 & 0,02 \\
\hline 88 & 8,0 & 323 & 145 & 180 & 155 & 15,0 & 5,2 & 0,70 & 0,01 & 1,48 & 0,04 & 0,01 \\
\hline A9 & 7,9 & 351 & 175 & 210 & 185 & 10,0 & 8,2 & 0,72 & $\operatorname{tr}$ & 0,60 & 0,00 & 0,02 \\
\hline A10 & 7,9 & 530 & 200 & 275 & 190 & 17,5 & 0,2 & 0,37 & 0,01 & 5,52 & 0,00 & 0,02 \\
\hline 89 & 7,9 & 560 & 180 & 225 & 165 & 17,0 & 4,0 & 0,50 & 0,01 & 2,04 & 0,02 & 0,01 \\
\hline Al1 & 8,0 & 381 & 180 & 230 & 190 & 12,5 & 6,4 & 0,72 & tr. & 0,54 & 0,00 & 0,02 \\
\hline A12 & 7,9 & 358 & 185 & 195 & 190 & 12,5 & 1,8 & 0,62 & tr. & 0,64 & 0,00 & 0,02 \\
\hline$A 13$ & 7,9 & 448 & 200 & 225 & 190 & 12,5 & 1,2 & 0,50 & tr. & 1,80 & 0,00 & 0,02 \\
\hline $\mathrm{B} 10$ & 7,8 & 418 & 165 & 195 & 165 & 32,5 & 5,2 & 0,45 & 0,01 & 2,0 & 0,03 & 0,02 \\
\hline B11 & 7,8 & 400 & 145 & 185 & 165 & 27,5 & 3,6 & 0,40 & 0,01 & 2,12 & 0,02 & 0,02 \\
\hline Al4 & 8,0 & 425 & 170 & 215 & 170 & 32,5 & 19,0 & 1,00 & 0,01 & 2,06 & 0,13 & 0,15 \\
\hline$B 12$ & 7,9 & 410 & 160 & 185 & 165 & 30,0 & 18,2 & 0,72 & 0,01 & 2,08 & 0,38 & 0,01 \\
\hline B13 & 7,9 & 412 & 155 & 170 & 170 & 27,5 & 5,0 & 0,55 & tr. & 2,06 & 0,05 & 0,05 \\
\hline A15 & 8,0 & 358 & 165 & 190 & 165 & 12,5 & 1,4 & 0,47 & tr. & 0,64 & 0,00 & 0,03 \\
\hline B14 & 7,9 & 398 & 160 & 190 & 165 & 22,5 & 2,6 & 0,60 & tr. & 1,84 & 0,03 & 0,01 \\
\hline $\mathrm{A} 16$ & 8,1 & 355 & 150 & 180 & 145 & 10,0 & 6,6 & 0,50 & $\operatorname{tr}$ & 1,64 & 0,02 & 0,03 \\
\hline$B 15$ & 7,9 & 413 & 165 & 195 & 165 & 30,0 & 1,8 & 0,47 & 0,01 & 2,18 & 0,02 & 0,01 \\
\hline
\end{tabular}




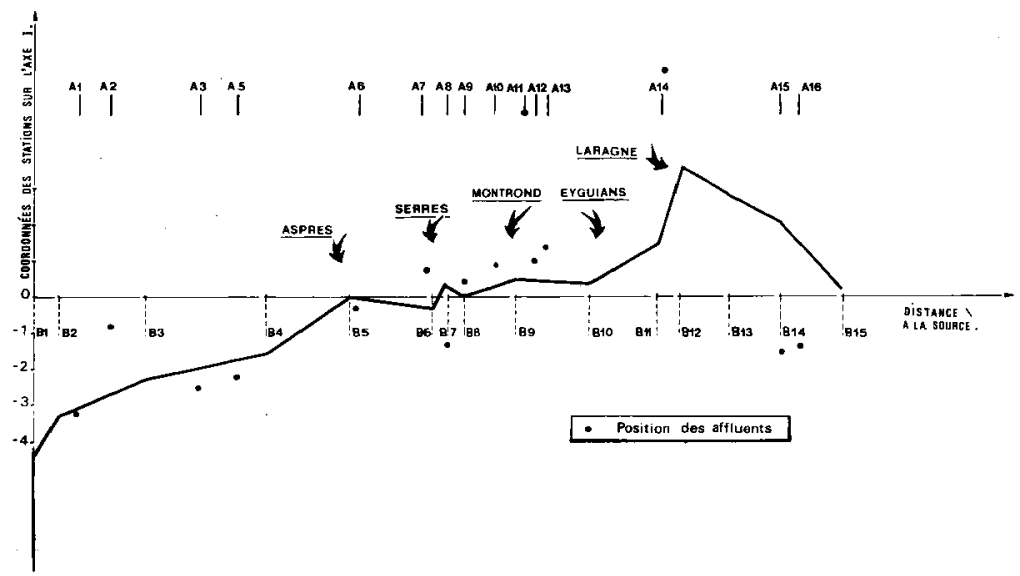

Fig. 9 : Evolution de l'amont vers l'aval de la minéralisation des eaux du Buëch en septembre, codée par les coordonnées des stations sur le premier axe d'une A.C.P. portant sur les paramètres chimiques.

concentrations " normales" du secteur en cause. Cette perturbation des conditions naturelles, imputables aux rejets domestiques ou aux activités agricoles et industrielles, correspond à une eutrophica. tion du milieu.

Les deux processus d'eutrophisation et d'eutrophication bien mis, ici, en évidence par l'A.C.P., sont fondamentalement différents; ils ne sont cependant pas toujours faciles à discerner l'un de l'autre puisqu'ils se manifestent souvent de façon analogue (Fabri 1984); ceci explique la contribution importante des descripteurs de "pollution ${ }^{1}$ ou d'eutrophisation sur les deux axes.

\section{Travaux cités}

Dajoz (R.) 1972. Précis d'écologie. Paris, Dunod, 434 p.

Doledec (S.) 1986. Les peuplements de macroinvertébrés benthiques du cours inférieur de l'Ardèche. Dynamique spatiotemporelle. These de 3eme cycle, Lyon I. 246 p.

1. Le concept de * pollution * est employé dans le même sens que * eutrophication *, en faisant référence à l'environnement naturel étudié laissant au terme * eutrophisation » le sens défini par Duvigneaud (1974) et Dajoz (1977).
Duvigneaud (P.) 1974. La synthèse écologique. Doin, Ed., Paris. $296 \mathrm{p}$.

Fabri (R.) 1984. Etude écologique des rivières du nord du massif ardennais (Belgique): flore et végétation de Diatomées et physico-chimie des eaux. Thèse de Doctorat, Université de Liège. $201 \mathrm{p}$.

Giudicelli (J.), Dia (A.) \& Légier (P.) 1980. Etude hydrobiologique de l'Argens (Var) : peuplements invertébrés et distribution longitudinale. Bijdr. Dierkd., $50: 303-341$.

Guilbot (A.) 1981. Etude de la qualité des eaux de rivière du bassin du Rhône-Méditerranée-Corse. Note de synthèse. Agence de bassin Rhôtle-Méditerranée-Corse, Pierre-Bénite, 60310, France. $23 p$.

Guilbot (A.), Picot-Reboul (B.), Bouchaud (M.) \& Roux (M.) 1982. Etude de l'évolution de la qualité des eaux de rivière. Water Res., 16:1173-1187.

Klein (L.) 1960. Aspects of river Pollation. Butterworth ed., London, $621 \mathrm{p}$.

Landreau (A.) 1974. Etude statistique de la pollution de la Seine en 1971. Analyse ou composantes principales. T.S.M. L'Eats, 69, $3: 129-138$.

Martin (G.) 1979. Le probleme de l'azote dans l'eau. Technique et documentation, Paris. 279 p.

Nisbet (M.) \& Verneaux (J.) 1970. Composantes chimiques des eaux courantes. Discussion et proposition de classes en tant que bases d'interprétation des analyses chimiques. Annls Limnol., $6(2): 161-190$ 